\title{
Troubleshooting intraoperative complications of penile prosthesis placement
}

\author{
Devang Sharma, Ryan P. Smith \\ Department of Urology, University of Virginia Health System, Charlottesville, VA 22908, USA \\ Contributions: (I) Conception and design: D Sharma, RP Smith; (II) Administrative support: None; (III) Provision of study material or patients: None; \\ (IV) Collection and assembly of data: D Sharma; (V) Data analysis and interpretation: D Sharma; (VI) Manuscript writing: All authors; (VII) Final \\ approval of manuscript: All authors \\ Correspondence to: Ryan P. Smith, MD. Department of Urology, University of Virginia Health System, PO Box 800422, Charlottesville, VA 22908, USA. \\ Email: rps2k@virginia.edu.
}

\begin{abstract}
Placement of a penile prosthesis is an excellent option for well-selected patients, offering high patient satisfaction and a low rate of complications. Most urologists who perform the procedure are not high volume implanters and may have limited experience with troubleshooting intraoperative issues. In this review, the authors use current literature to provide recommendations for the management of various intraoperative issues such as difficultly with dilation, incongruent measurement, perforation, urethral injury, crossover, complications of reservoir placement, penile curvature, glans mobility, and hemostasis are all discussed. Preparedness for the management of intraoperative issues can help improve patient outcomes and limit morbidity.
\end{abstract}

Keywords: Erectile dysfunction; intraoperative complications; penile prosthesis

Submitted Jul 04, 2017. Accepted for publication Jul 06, 2017.

doi: $10.21037 /$ tau.2017.07.13

View this article at: http://dx.doi.org/10.21037/tau.2017.07.13

\section{Introduction}

Placement of a penile prosthesis is an excellent option for the motivated and well-informed patient with medically refractory erectile dysfunction. The surgery offers high patient satisfaction and has a low rate of complications, especially in the hands of an experienced surgeon. Yet, urologists who perform fewer than five implants per year are responsible for $75 \%$ of the penile prostheses in the United States (1). In the United Kingdom, $80 \%$ of surgeons who perform the procedure only place one or two penile prostheses per year (2). The placement of penile prostheses is thus a low volume operation for most urologists who perform the procedure.

The relationship between patient outcomes and surgical volume is well documented for a variety of surgeries and holds true for penile implantation as well $(3,4)$. Increasing awareness of various intraoperative problems and preparedness for troubleshooting is of critical importance, as many urologists who place penile prostheses do not have the benefit of high surgical volume.

In this review, the authors present a guide to troubleshooting several of the most common intraoperative problems in the placement of the penile prostheses. A review of current literature, including expert opinion and various study designs, was used to complete this comprehensive analysis.

\section{Discussion}

Prior to delving into troubleshooting of intraoperative issues, it should be noted that the best approach to complications is always prevention. Careful preparation and understanding of potential issues is critical. The prevention of post-operative complications such as infection and erosion are a separate topic and will not be discussed in this review. Low volume implanters should consider referring 
challenging cases to high volume centers of excellence. The ensuing discussion follows the steps for placement of an inflatable penile prosthesis.

\section{Dilation}

After obtaining adequate exposure of the corpora, corporotomies are made to allow for dilation. Issues with corporal dilation may be related to the incision chosen by the surgeon. While both transscrotal and infrapubic approaches are highly effective, corporal dilation may be easier from a transscrotal approach. Trost et al. reported approximately $1.5 \mathrm{~cm}$ longer proximal dilation in high volume prosthesis implanters across multiple institutions in 2012 (5). While distal measurements were not different between the two approaches, excessive abdominal girth can make distal dilation from an infrapubic approach more challenging. Ultimately, the approach is left to the discretion of the surgeon and is often dictated by training and familiarity. Regardless of approach, dilators should always be angled dorsolaterally to avoid the urethra during distal dilation and follow the crura during proximal dilation.

If the angle of approach seems appropriate but the corpora are difficult to dilate, the patient may suffer from corporal fibrosis. This can be secondary to diabetes, intracavernosal injection therapy for erectile dysfunction, Peyronie's disease or injection therapy for its treatment, prolonged ischemic priapism, prior prosthesis infection, or trauma (6). It is helpful to consider the minimal diameter required for the cylinders available for implantation. The AMS 700 CXR cylinders only require dilation to $9 \mathrm{~mm}$. Better erection quality may be achieved with wider cylinders, so this should also be considered in cylinder selection and attempts at dilation.

If corporal fibrosis is expected, the location of the corporotomies can be selected to provide easier dilation. If scarring from a prior transscrotal implant placement is suspected, an infrapubic approach is preferred. If other etiologies of fibrosis are expected, mid-corporal corporotomies are helpful to minimize the length of dilation required in a single direction. For example, a penoscrotal incision may be chosen over a perineal incision or an infrapubic incision over a subcoronal incision (7).

Manufacturer provided dilators may be insufficient for fibrotic corpora. Dilation can be attempted with Hegar, Brooks, or Uramix dilators, with the later being the most useful for fibrosis (8). The Uramix or Moorevillle dilators were developed to allow for drilling of a cavity by serially passing cutting dilators into the corpora. A twisting or oscillating movement is used with these instruments. A higher rate of distal and proximal perforation may be encountered with their use, although this could be attributed to the higher difficulty associated with these cases (9).

Metzenbaum scissors may also be used to aid in dilation or for excavation of corporal tissue. Tips should always point laterally and spreading is preferable to cutting. An advanced technique described by Kramer, et al., uses dilation in shorter segments by alternating use of the Metzenbaum scissors with a Dilamezinsert (10). Counter incisions or additional corporotomies may also be helpful to complete more distal dilations.

In cases of difficult dilation, other intraoperative issues such as perforation, urethral injury, and crossover may occur. These are discussed in future sections below.

\section{Measurements}

Once the corpora are dilated distally and proximally, measurements allow for correct cylinder selection. It is generally recommended that the same cylinder length be used in each corpus for maximal symmetry. In situations where the corpora measure within $1 \mathrm{~cm}$ of each other, the shorter of the two measurements should be used to minimize pain and chance of erosion. This level of variation is not clinically significant and should not be problematic. If one measurement is much longer than expected, it may represent a proximal perforation. If the discrepancy is subtler, it could be a crossover.

Sometimes the measurements are similar in total length but the distal lengths vary. There may be asymmetry in how far the dilators extend distally. It is critical to ensure a distal urethral injury has not occurred. If this is absent, the distal discrepancy is more likely to represent anatomic variation. The corpora do not always extend an equal distance into the glans. Capsule formation may offset this variation once developed in the postoperative course.

\section{Perforation and urethral injury}

Perforation can occur during dilation in either direction. Some indicators of perforation include discrepancy in measurements, sudden loss of resistance during dilation, penile edema during distal corporal irrigation, or migration of the distal cylinder tips to the more proximal shaft. Distal and proximal perforations are managed differently.

Distal perforations necessitate careful assessed for urethral 
injury. Inspection may show extrusion through the meatus, blood at the meatus, or a "distal fluid challenge test" may show irrigation fluid passing around the urethral catheter. The safest course is to abort the procedure and address the urethral injury. If the implant is completed, the urethral injury increases the risk of implant infection and the cylinders impair healing of the urethral injury. Patient with diabetes or those who require intermittent catheterization are at even higher risk for issues with wound healing (6).

A distal urethral injury should be inspected and assessed for the need for a primary closure. Small distal urethral injuries may heal without repair. A catheter can be placed, but prolonged catheter placement is not necessary. The urinary stream will preferentially draw fluid into itself and out of the urethra rather than infiltrate the corporal defect (7). Prolonged catheter placement may even increase the risk of stricture.

For larger injuries that require repair, the key is adequate exposure for the two-layer closure. If a distal urethral injury cannot be accessed through the meatus, creation of an iatrogenic hypospadias may be necessary. Proximal urethral injuries, especially in the penoscrotal aspect of the urethra, are readily accessible from a transscrotal approach (6).

There have been reports of the feasibility of single cylinder placement in unilateral urethral injuries that can be adequately repaired (6). The authors would argue that this should rarely be considered and only by an experienced implanter. Patient satisfaction with a single inflatable cylinder is considerably lower than with two-cylinder placement. With the increasing prevalence of obesity, a greater number of patients have diabetes and are at higher risk for impaired wound healing and infection. A nondiabetic, non-smoker who has a well-closed unilateral urethral injury and is undergoing malleable prosthesis placement would be the ideal candidate for consideration of unilateral cylinder placement.

Corporal perforations without urethra injury allow for safe completion of the implantation. A distal perforation will heal without repair if the corpora can be redilated and the cylinder placed appropriately. A proximal perforation should be addressed with the creation of a sling to prevent proximal migration of the cylinder. This is described well by Mulcahy (7). First, a double swedged permanent suture is placed through the proximal cylinder or rear tip extender. Next, the cylinder is placed and the suture is anchored to the lateral and medial aspect of the corporotomy distal to the exit of the input tubing. The corporotomy is closed primarily, and the sling suture is tied above the closure.
Cutting the permanent suture of the sling two to three centimeters from the knot allows for easier identification and removal for cylinder replacement in the future, if needed (7). Detailed understanding of this technique will allow for confident completion of implantations complicated by proximal perforations.

\section{Crossover}

Crossover is another issue related to corporal dilation. Using the appropriate dorsolateral angle when performing corporal dilation is the best approach to preventing crossover. Indicators include contact during simultaneous dilation, unequal corporal measurements, difficulty with placement of the second cylinder, and a lopsided, thin, or otherwise unusual appearance of the erection during inflation (6). Crossover is a manageable intraoperative issue.

First, the dilator is placed in the common cavity, the side that "received" the crossover. Next, the contralateral side is redilated using a more lateral trajectory. The first cylinder is placed in this newly dilated tract, maintaining the dilator in the contralateral cavity to prevent erroneous cylinder placement. Once the first cylinder is appropriately placed, the dilator is removed from the contralateral corpora and is replaced by the second cylinder (6).

\section{Reservoir placement and intra-abdominal injuries}

Once the corpora are appropriately dilated, reservoir placement typically precedes cylinder placement (8). The traditional location for reservoir placement is the space of Retzius. While rare, there have been several reports of complications related to this approach including bladder perforation, avulsion of a branch of the external iliac vein, postoperative venous compression syndrome, herniation of the reservoir, and direct inguinal herniation of intraabdominal contents (11). Cadaveric studies have shown the bladder and external iliac vessels can be as close as two centimeters from the inguinal ring, which is closer than previously appreciated (12). Certain patient factors such as prior robotic-assisted laparoscopic prostatectomy or radical cystectomy can further complicate the anatomy by violating the space of Retzius. Scarring can be present from other prior abdominal surgeries, pelvic radiation, or from urinoma after anastomotic leak of a urethral anastomosis (7). This can lead to more complications with reservoir placement, especially among inexperienced surgeons. Some have defaulted to ectopic reservoir placement for patients with histories of 
robotic prostatectomy for this reason (13). Others have used ectopic reservoir placement routinely (14).

Central goals to favorable reservoir placement include preventing the patient from seeing or feeling the reservoir, preventing autoinflation, erosion and extrusion, and limiting the rare complications reported above. Ectopic placement may offer fewer complications when compared to traditional placement in the space of Retzius. Ectopic placement is typically in one of two locations. Most commonly, the reservoir is placed between the transversus abdominis anteriorly and the transversalis fascia posteriorly. In more medial placement, it may be placed between the rectus abdominis muscle anteriorly and transversalis fascia posteriorly. An advantage of traditional placement over ectopic placement is that it is offers a less conspicuous location for the patient. However this is less of an issue in obese patients due to the increased adiposity of their anterior abdominal walls. In fact, an ectopic location anterior to the muscles of the abdominal wall has been reported in a morbidly obese patient with good surgical and functional outcome (11). With the prevalence of robotic prostatectomy and obesity among patients being considered for penile prosthesis placement, surgeons must be comfortable with ectopic reservoir placement. This should help prevent intraoperative complications related to reservoir placement.

When traditional reservoir placement is preferred, a technique presented by Levine and Hoeh can help avoid creating inguinal floor weakness and visceral injury (11). Curved Jorgensen scissors are placed through the operative incision and over the pubis with the tips pointing posteriorly. By lifting the scissors and using the pubic as a fulcrum, the transversalis fascia is sharply perforated allowing for entry and dissection of the space of Retzius (11). The authors acknowledge that the approach to reservoir placement is one of the most variable steps of penile prosthesis placement. It is advisable to be proficient in one or two techniques and be familiar with others.

If visceral or vascular injury is suspected during any part of reservoir placement and filling, the first step is to ensure adequate exposure. Either the original incision should be extended or a new incision made. The appropriate intraoperative consultation should be made to help assess and manage any bowel or vascular injury. Hematuria is an indicator of possible bladder injury. In this instance, the space of Retzius should be packed and the bladder filled with a mixture of saline and methylene blue (8). Dye on the packing is a clear indicator of a bladder injury, for which exposure, two-layered closure, and either ectopic reservoir placement or aborting the implantation is recommended.

\section{Cylinder placement, testing, and closure}

After corporal dilation and reservoir placement, the cylinders are ready to be placed. A good dilation should ensure smooth cylinder placement. With the components of the prosthesis in place, they are connected and cycled to test the system. This is where additional issues such as penile curvature or glans hypermobility may be discovered.

If penile curvature is noted during the inflation of the cylinders, the approach to address this depends on the etiology and degree of curvature. If a distal lateral curvature is noted, a crossover may have occurred. This can be assessed with cylinder removal and simultaneous placement of metal dilators, which should not touch each other. In the absence of crossover, a curvature of 20 degrees or less does not require correction as the rigidity of the device should provide reasonable functional outcome (6). The cylinder selection should be considered in this situation. Cylinders that expand in the length and girth may be less favorable in patients who have penile curvature. For these patients, consideration should be given to using wider cylinders that expands only in girth.

For higher degrees of curvature, the first step should be penile modeling. This is done by fully inflating the cylinders and placing a rubber shod on the tubing to protect the pump. The penis is forcefully bent 90 degrees opposite the point of maximum curvature and held in place for 90 seconds. If curvature persists, the clamps are removed and more fluid is added to the cylinders for another round of modeling. This approach should remedy curvature caused by Peyronie's disease in most cases (7). If the curvature is as a result of scarring from prior infected prosthesis, adjuvant procedures such as plication or excision with or without grafting should be performed at the time of implantation (6).

With the cylinders fully inflated, the glans may be noted to be hypermobile or floppy. This can occur in any direction. When it occurs ventrally, it is sometimes called the supersonic transport (SST) deformity for its resemblance to that type of aircraft. Glans hypermobility is more common in uncircumcised patients (7). In mild cases, the foreskin can be retracted to provide glans stability adequate for penetration. If the defect seems more pronounced, it is important to ensure the cylinder length is appropriate. Tilting due to hypermobility and tilting due to short cylinders has a different appearance. 
Surgical correction can be considered, but this should be weighed against the risk of injury to the neurovascular bundle and distal cylinder tips. To correct the defect, a hemi-circumscribing incision is made opposite the direction of tilting. The glans is dissected from the distal tips of the corpora with the cylinders inflated. Care is taken to avoid the neurovascular bundle and the cylinders. Permanent suture is placed in the glans tissue using a large curved needle and anchored to the distal tunica albuginea, tying the sutures after each suture is placed to facilitate exposure (7).

Once a satisfactory erection is created, the corporotomies should be closed. Watertight closure of the corporotomies is unnecessary and increases the risk of iatrogenic injury to the cylinders with each needle pass. Stay sutures on either side of a corporotomy can be tied to each other to close the corporotomies and capsule formation will further seal the sites of entry. In situations where bleeding is encountered, various strategies can be used to reduce the risk of postoperative hematoma. Sadeghi-Nejad et al. showed the efficacy of one strategy using a closed suction drain and pressure dressing (15). A 10 French Round Blake (Johnson \& Johnson) is placed, the cylinders are left approximately $70 \%$ inflated, and a pressure dressing is applied. The following day, the drain is removed, the cylinders deflated, and the dressing taken down. In their series of over 400 patients from multiple surgeons and sites, the rate of scrotal hematoma was less than $1 \%$, and the rate of infection was about $3 \%$ at 18 months follow-up (15). Closed suction drain placement is a safe option to consider for surgeons who do not regularly use them with penile prostheses.

\section{Conclusions}

Surgeons will invariably encounter complications during penile prosthesis placement in their careers. Intraoperative issues and complications require experience and sound judgment to limit patient morbidity. Awareness and preparedness for managing various intraoperative issues is critical, especially for surgeons who perform the procedure infrequently. As the demand for urologists performing penile prosthesis surgery increases, preparedness should help ensure the procedure continues to provide a low rate of complications and high patient satisfaction.

\section{Acknowledgements}

None.

\section{Footnote}

Conflicts of Interest: The authors have no conflicts of interest to declare.

\section{References}

1. Oberlin DT, Matulewicz RS, Bachrach L, et al. National practice patterns of treatment of erectile dysfunction with penile prosthesis implantation. J Urol 2015;193:2040-4.

2. Agrawal V, Ralph D. An audit of implanted penile prosthesis in the UK. BJU Int 2006;98:393-5.

3. Henry GD, Kansal NS, Callaway M, et al. Centers of excellence concept and penile prosthesis: An outcome analysis. J Urol 2009:181:1264-8.

4. Lotan Y, Roehrborn CG, McConnell JD, et al. Factors influencing the outcomes of penile prosthesis surgery at a teaching institution. Urol 2003;62:918-21.

5. Trost LW, Boonjindasup AG, Hellstrom WJ. Comparison of infrapubic versus transscrotal approaches for inflatable penile prosthesis placement: a multi-institutional report. Int J Impot Res 2015;27:86-9.

6. Levine LA, Becher E, Bella A, et al. Penile prosthesis surgery: Current recommendations from the international consultation on sexual medicine. J Sex Med 2016;13:489-518.

7. Mulcahy JJ. The prevention and management of noninfectious complications of penile implants. Sex Med Rev 2015;3:203-13.

8. Pathak RA, Broderick GA. Inflatable penile prosthesis implantation. In: Smith JA, Howards SS, Preminger GM, et al. editors. Hinman's Atlas of Urologic Surgery, 4th Edition. Elsevier, Inc., Philadelphia, 2018:890-9.

9. Mooreville M, Adrian S, Delk JR 2nd, et al. Implantation of inflatable penile prosthesis in patients with severe corporal fibrosis: Introduction of a new penile cavernotome. J Urol 1999;162:2054-7.

10. Kramer AC, Goldmark ER, Reznicek D. Placement of inflatable penile prosthesis with severe corporal fibrosis from priapism. J Sex Med 2014;11:2367-70.

11. Levine LA, Hoeh MP. Review of penile prosthesis reservoir: Complications and presentation of a modified reservoir placement technique. J Sex Med 2012;9:2759-69.

12. Henry G, Hsaio W, Karpman E, et al. A guide for inflatable penile prosthesis reservoir placement: Pertinent anatomical measurements of the retropubic space. J Sex Med 2014;11:273-8.

13. Kohler TS, Moore A, Ring J. A contemporary series 
of penile implant complications occurring at a training institution. J Sex Med 2016;13:S68.

14. Kavoussi NL, Hofer MD, Viers BR, et al. Synchronous ipsilateral high submuscular placement of prosthetic balloon and reservoirs. J Sex Med 2017;14:64-8.

Cite this article as: Sharma D, Smith RP. Troubleshooting intraoperative complications of penile prosthesis placement. Transl Androl Urol 2017;6(Suppl 5):S892-S897. doi: 10.21037/ tau.2017.07.13
15. Sadeghi-Nejad H, Ilbeigi P, Wilson SK, et al. Multiinstitutional outcome study on the efficacy of closedsuction drainage of the scrotum in the three-piece inflatable penile prosthesis surgery. Int J Impot Res 2005;17:535-8. 